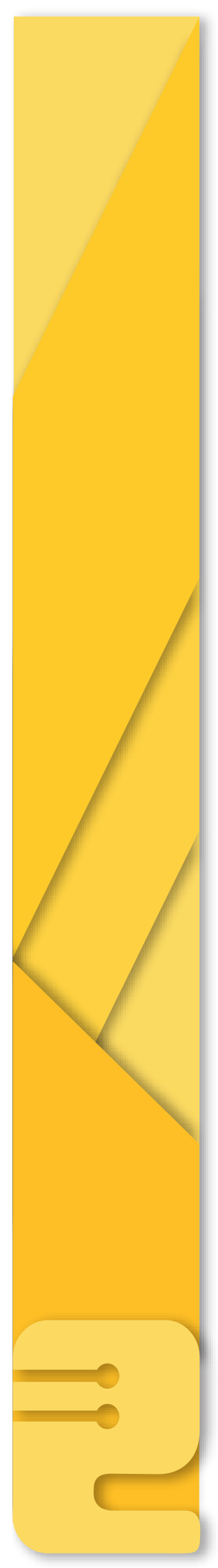

\title{
ANÁLISIS DEL MOVIMIENTO MEDIANTE LA FOTOGRAFÍA ESTROBOSCÓPICA
}

\author{
MOTION ANALYSIS USING STROBE PHOTOGRAPHY \\ ANÁLISE DO MOVIMENTO MEDIANTE A FOTOGRAFIA ESTROBOSCÓPICA
}

Juan Gabriel Martínez Alméciga ${ }^{1}$, Diana Mayerly Galván Padilla²

Fecha de recibido: 1 de febrero de 2016 | Fecha de aprobado: 25 de Marzo de 2016

\section{Resumen}

Un método para la adquisición de imágenes estroboscópicas mediante el uso de un estroboscopio mecánico de bajo presupuesto, se utilizó en el estudio del movimiento rectilíneo uniforme, uniformemente acelerado y movimiento en dos dimensiones de corta duración. Por medio de la utilización de software libre y de código abierto se realizó el análisis de las fotografías estroboscópicas obteniendo las ecuaciones que describen cada uno de los movimientos y el valor de la aceleración de la gravedad para la ciudad de Bogotá, Colombia.

Palabras clave: Fotografía estroboscópica, movimiento uniformemente acelerado, movimiento en dos dimensiones, estroboscopio mecánico, enseñanza de la física.

1 Magíster en Ciencias-Física. Licenciado en Física. Docente de tiempo completo de La Corporación Universitaria Minuto de Dios - UNIMINUTO. Integrante del grupo de investigación "Investiciencias" - UNIMINUTO. Correo electrónico: jumartinez@uniminuto.edu

2 Estudiante de Ingeniería Civil. Integrante del semillero de Física (2013-2015). Corporación Universitaria Minuto de Dios - UNIMINUTO. 


\section{Abstract}

We used a method for the acquisition of stroboscopic images by using a economical mechanical stroboscope. It was used in the study of rectilinear uniform motion, uniformly accelerated motion and short duration 2-dimensional motion. The analysis of each strobe photograph was performed using free and open source software. Motion equations describing each of the movements and the gravity acceleration value for Bogota city were obtained.

Keywords: Stroboscopic photography, uniformly accelerated motion, two dimensional motion, strobe, physics education.

\section{Resumo}

Um método para a aquisição de imagens estroboscópicas mediante o uso de um estroboscopio mecânico de baixo orçamento, utilizou-se no estudo do movimento rectilíneo uniforme, uniformemente acelerado e movimento em duas dimensões de curta duração. Por médio da utilização de software livre e de código aberto realizou-se a análise das fotografias estroboscópicas obtendo as equações que descrevem a cada um dos movimentos e o valor da aceleração da gravidade para a cidade de Bogotá, Colômbia.

Palavras-chave: Fotografia estroboscópica, movimento uniformemente acelerado, movimento em duas dimensões, estroboscopio mecânico, ensino da física. 


\section{INTRODUCCIÓN}

El empleo de la fotografía estroboscópica para el estudio del movimiento dejó de ser una técnica utilizada por laboratorios de investigación en la década de los 60 del siglo pasado. Ésta pasó a convertirse en una herramienta novedosa en la enseñanza de las ciencias, específicamente, de la física, (Ritchie, 1966). No obstante, la aplicación de la técnica en los laboratorios escolares de aquél entonces estaba condicionada a la adquisición de un equipo de fotografía, una lámpara estroboscópica y a los dispositivos requeridos para realizar el revelado y posterior positivado de las películas fotosensibles utilizadas en la época. Aunque con la incorporación de las cámaras Polaroid se minimizaba el proceso y tiempo de revelado y positivado, se incrementaban los costos para los laboratorios escolares (Dorling, 1966).

El advenimiento de la fotografía y del vídeo digital permitieron superar inconvenientes de décadas pasadas y se han convertido en una herramienta imprescindible, tanto para la investigación científica como para la enseñanza de las ciencias (Valdés, 2007; Medeiros, 2009). Una de las aplicaciones importantes de la fotografía y el vídeo digital tiene que ver con el estudio de fenómenos de corta duración, como es el caso de la caída de objetos y disparos de proyectil, estudiados en los cursos introductorios de física mecánica. De igual manera, con el estudio de la rotura de objetos debido a impactos y movimientos del cuerpo humano (Laws, 1998; Molina, 2006).

Una de las técnicas comunes en la producción de fotografías estroboscópicas precisa la utilización de una lámpara que emita destellos de luz consecutivos $y$ de frecuencia regulable. Cuando un objeto se encuentra en movimiento en un recinto oscuro y sólo es iluminado por este tipo de lámpara, cada vez que se genera un destello toda la escena parece congelada, pero con el objeto en una posición diferente. Al registrar esto, por medio de una cámara fotográfica en una sola toma y durante un tiempo adecuado, se obtiene una fotografía estroboscópica.

Algunos autores utilizan vídeos cortos del objeto en movimiento y con un software de edición extraen los fotogramas y los convierten en archivos de imagen que posteriormente editan en una sola imagen, dando como resultado una fotografía estroboscópica (Madeiros, 2009).

A diferencia de los métodos mencionados para la adquisición de las imágenes de cuerpos en movimiento y eventos de corta duración, en este trabajo no se utilizan vídeos y se prescinde de la lámpara estroboscópica. Las fotografías se obtienen mediante el uso de un estroboscopio mecánico y otros elementos de fácil adquisición en laboratorios escolares.

El estroboscopio es un dispositivo que permite observar de manera periódica un fenómeno, (Colombo, 1992). En su forma básica, el estroboscopio mecánico consiste principalmente en un disco con una o más ranuras separadas igualmente una de la otra y dispuesto sobre un eje de rotación y un soporte (ver figura 1).

Figura 1. Estroboscopio mecánico con disco de acetato de doble ranura y soporte en MDF
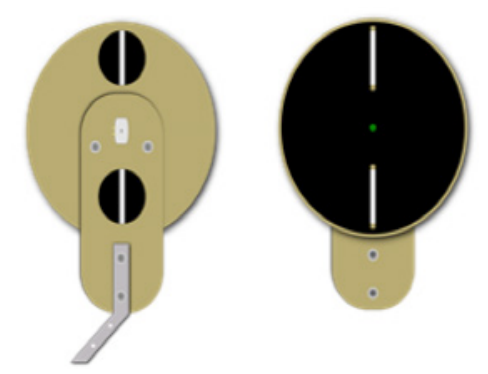

Fuente: adaptación de los autores.

Por otra parte, cuando un objeto se encuentra en movimiento y se desea fotografiar, dependiendo de las condiciones de luminosidad, es necesario modificar en la cámara el tiempo de apertura del diafragma (que actúa de manera semejante al iris del ojo humano), para que entre la luz necesaria y no se pierda detalle alguno de la imagen. Si la cámara permanece en reposo y el tiempo de exposición es largo, la imagen capturada por el sensor de la cámara se presentará como una estela borrosa. Este efecto es consecuencia del registro continuo de la información proveniente del objeto en movimiento. Ahora, si delante de la cámara se coloca el estroboscopio y se permite que gire, la luz que incide en el sensor está limitada a la que atraviese en ese instante una ranura del disco, que se encuentre enfrente del sistema de lentes que forman el objetivo. Como la rapidez de giro se mantiene constante, se registran en una sola toma diferentes posiciones del objeto en los mismos intervalos de tiempo; siempre y cuando, el objeto permanezca en el campo visual de la cámara y el tiempo que dure su movimiento sea mayor al que tarda en que una nueva ranura aparezca en el objetivo. De esta manera se obtiene un fotografía estroboscópica. 
El desarrollo de este trabajo surgió a partir del interés de los integrantes del semillero de investigación de Física de estudiar eventos de corta duración en cursos introductorios de física mecánica.

\section{MONTAJE EXPERIMENTAL}

Para estudiar los movimientos uniformes (мU), uniformemente acelerado (MUA) y el movimiento en dos dimensiones, se realizaron tres montajes diferentes. En cada uno de ellos se utilizó una cámara fotográfica Canon EOS Rebel i en modo manual, posibilitando el control del tiempo de apertura del diafragma y la sensibilidad iso del sensor. Se trabajó con un lente objetivo EF-S 1- $55 \mathrm{~mm}$ de la misma casa fabricante. El tiempo de apertura del diafragma se fijó entre 0.6 s y $1.0 \mathrm{~s}$, dependiendo del movimiento a estudiar, y la sensibilidad a 400 ISO, con una apertura de diafragma de $f / 5.0$ y $f / 5.6$, permitiendo que ingresara la mayor cantidad de luz posible para longitudes focales de $35 \mathrm{~mm}$ y $55 \mathrm{~mm}$ del lente-objetivo, respectivamente. El sistema cámara-estroboscopio se montó sobre un trípode de $1.0 \mathrm{~m}$ de alto. Para evitar algún tipo de movimiento brusco durante las fotografías se adaptó a la cámara un disparador accionado de manera remota. Cada una de las tomas fotográficas fueron realizadas sólo con luz ambiente. En todos los montajes se fijaron una o dos reglas en el plano donde se da el movimiento de los cuerpos a fotografiar, a fin de poder medir distancias y asignar coordenadas en el momento de realizar el respectivo análisis de las imágenes.

En el caso del mu y del MUA, en una superficie plana, se dispuso de un riel de 1.2 metros de longitud y sobre éste, un móvil de baja fricción al que previamente se le había ajustado un apuntador láser de color rojo. El riel se ubicó en un lugar paralelo a una pared de fondo negro, tal que, la señal luminosa del apuntador se observara sobre la pared en todo momento y así realizar las tomas fotográficas del punto de luz en diferentes posiciones. Para el MU, el móvil se impulsó valiéndose de la repulsión magnética entre dos imanes puestos en los extremos del riel y del móvil, y se dejó que rodara. El MUA se logró colgando en un extremo del móvil algunas pesas por medio de una cuerda pasando por una polea ubicada en un extremo del riel. El sistema cámara- estroboscopio se ubicó enfrente del riel a $3.5 \mathrm{~m}$ de distancia. Se utilizó un disco de una sola ranura y se permitió que girara a $(14.62 \pm 0.01) \mathrm{Hz}$.

El movimiento de caída libre se fotografío sobre un fondo negro dejando caer una pelota de golf desde una altura de $170.0 \mathrm{~cm}$. Se utilizó el estroboscopio con un disco de doble ranura. Por último, para el movimiento en dos dimensiones se realizaron lanzamientos de una esfera usando una rampa acanalada ubicada sobre una mesa a $90.0 \mathrm{~cm}$ del suelo. Se fotografío el momento en que la esfera salía despedida en la parte inferior de la rampa. En esta nueva disposición se fijaron dos reglas, ubicadas vertical y horizontalmente y se utilizó un disco con una ranura. En ambos casos la frecuencia de giro del disco fue de $(14.62 \pm 0.01) \mathrm{Hz}$. Las fotografías se realizaron sobre fondo negro.

La frecuencia de giro del disco y el tiempo entre ranuras se midió utilizando la señal de luz proveniente de un apuntador, ubicado en la parte posterior del sistema cámara-estroboscopio. Con cada giro del disco se permitió que la luz atravesara la ranura que estaba justo enfrente del puntero. La luz se registró por medio de un circuito con una fotorresistencia. La señal eléctrica generada en el circuito se leyó con la tarjeta de sonido de un PC y se analizó con el software de edición de audio Audacity. El tiempo de giro medido por Audacity se comparó con el obtenido por medio de un tacómetro digital marca DT-2234C.

Las fotografías fueron procesadas con software libre de edición de imágenes digitales Darktable en donde se recortaron y se variaron algunos atributos correspondientes a brillo-contraste y se dejaron en escalas de grises. En algunos casos se invirtieron los colores para que pudieran ser analizadas fácilmente. Las coordenadas de posición se asignaron a partir del análisis con el programa de gráficos vectoriales Inkscape. Los datos registrados fueron graficados y ajustados por mínimos cuadrados (Taylor, 1997) utilizando el software Qtiplot.

\section{RESULTADOS Y ANÁLISIS}

Las fotografías realizadas para el primer montaje se visualizan en la figura 2. En éstas se observan las diferentes posiciones del punto de luz sobre la pared. Los datos obtenidos de la figura 2 (a) se ajustaron a la ecuación de movimiento: 
Figura 2. Imágenes de señal luminosa de un puntero sobre fondo negro procesadas con Darktable. Tiempos de exposición 1.6 s. (a) Fotografía de cuerpo con movimiento uniforme. (b) Cuerpo con movimiento uniformemente acelerado

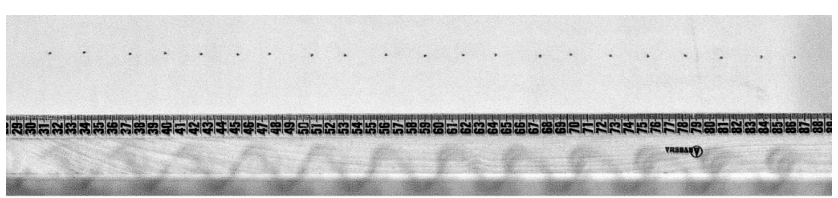

(a)

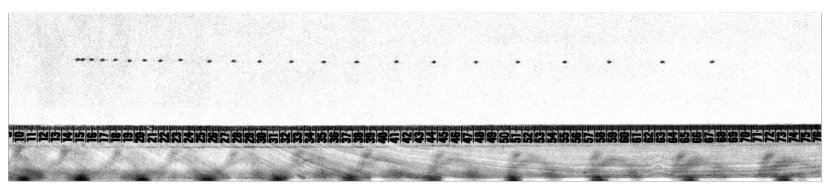

(b)

Fuente: adaptación de los autores.

$$
x=(31.00 \pm 0.13)+(40.0 \pm 0.7) t
$$

donde $x$ representa la posición medida en $\mathrm{cm}$ y $t$ el tiempo en $\mathrm{s}$, como se muestra en la figura 3(a). El ajuste corresponde a una línea recta, como se espera para un mu. La velocidad obtenida para el móvil fue de $(40.0 \pm 0.2) \mathrm{cm} / \mathrm{s}$ y la posición inicial de $(31.00 \pm 0.13) \mathrm{cm}$.

En el caso de la figura 2 (b), los datos fueron ajustados a la ecuación de movimiento:

Figura 3. (a) Datos experimentales y ajuste para el MU. (b) Datos experimentales y ajuste para el MUA. La posición x está medida en $\mathrm{cm}$ y el tiempo $\mathrm{t}$ en $\mathrm{s}$. Las respectivas ecuaciones fueron obtenidas a partir de un ajuste por mínimos cuadrados

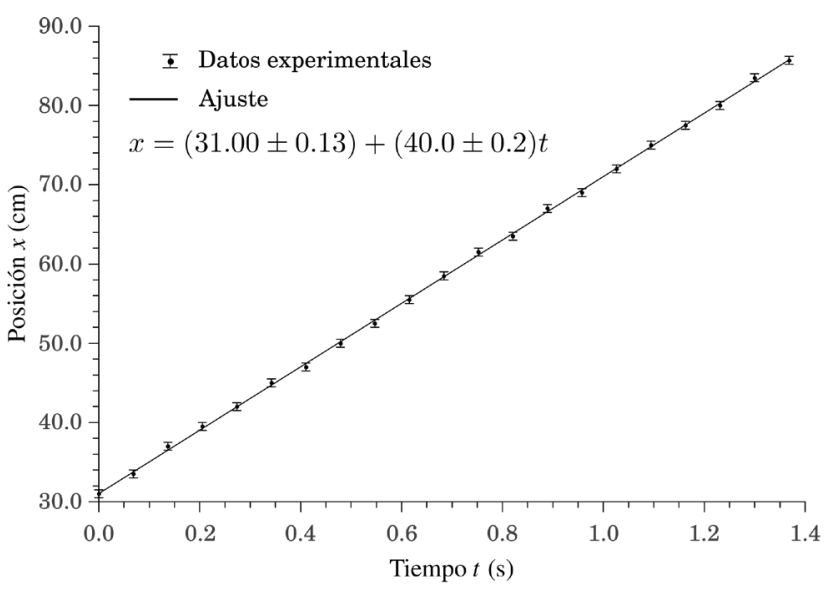

(a)

Fuente: adaptación de los autores.

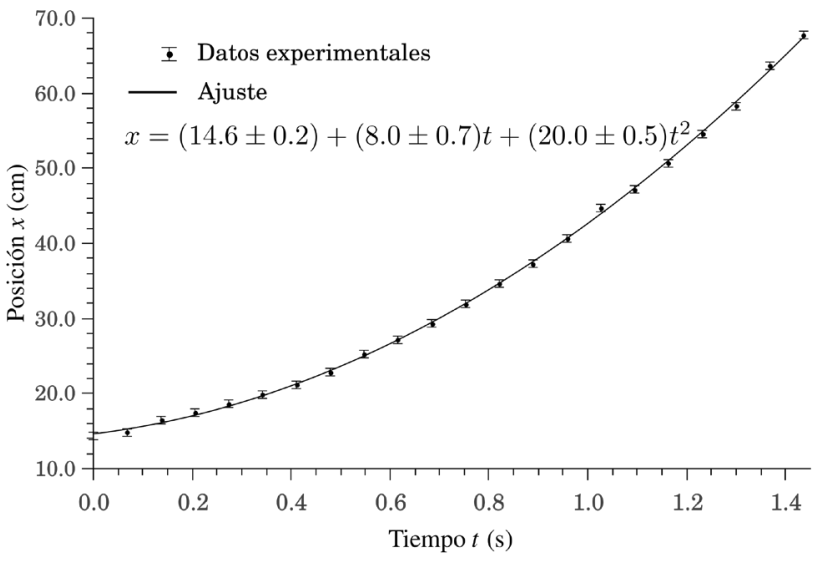

(b)

Fuente: adaptación de los autores.

$x=(14.65 \pm 0.2)+(8.0 \pm 0.7) t+(20.0 \pm 0.5) t^{2}$

El ajuste corresponde a una función cuadrática, como se observa en la Figura 3(b). De ésta se determinó que la posición inicial del móvil fue de la velocidad inicial de y la aceleración de La ec. (2) es propia de un MUA.

Por otra parte, la figura 4 muestra la imagen, en diferentes tiempos, de la posición de una bola de golf que está cayendo. Los datos obtenidos a partir de esta fotografía se ajustaron a la ecuación:

$$
y=-(4.57 \pm 0.07)+(129 \pm 1) t-(492 \pm 4) t^{2}
$$

En este caso $y$ representa la posición vertical en $\mathrm{cm}$ y $t$ el tiempo en $\mathrm{s}$.

Figura 4. Pelota de golf en caída libre. Se utilizó con el estroboscopio un disco de doble ranura

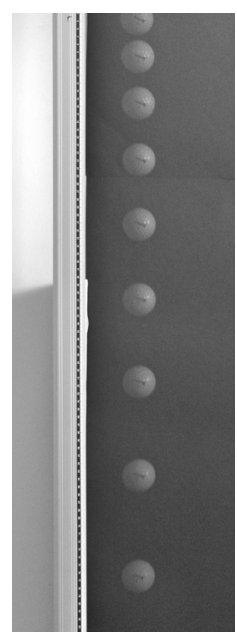

Fuente: adaptación de los autores. 
De la ec. (3) se encuentra que el valor de la aceleración de la gravedad obtenido a partir del ajuste es de $(984 \pm 8) \mathrm{cm} / \mathrm{s}^{2}$. Al comparar el anterior resultado con el reportado para la ciudad de Bogotá, Colombia de $977.39 \mathrm{~cm} / \mathrm{s}^{2}$ (Chanson, 2004), el valor encontrado experimental se puede considerar satisfactorio, dado que dentro del margen de incertidumbre contiene el valor aceptado.

Los datos experimentales y el ajuste correspondiente se relacionan en la figura 5 . El rango de tiempo con el que se realizó el análisis correspondiente fue inferior a $0.3 \mathrm{~s}$, lo que manifiesta la bondad del montaje propuesto para la captura de fotografías estroboscopicas, de eventos de corta duración.

En el último montaje se fotografío el movimiento de una esfera cuando sale por el extremo inferior de una rampa acanalada ubicada sobre una mesa, figura 6. En esta imagen se aprecia la trayectoria y las posiciones de la esfera a medida que va cayendo.

Las gráficas que muestran la posición contra el tiempo para la componente horizontal y vertical del movimiento semiparabólico se indican en la figura 7.

Figura 5. Datos experimentales y respectivo ajuste para la caída de una pelota. El valor experimental de la aceleración de la gravedad obtenido fue de $(984 \pm 8) \mathrm{cm} / \mathrm{s}^{2}$. El rango de tiempo fue menor a $0.3 \mathrm{~s}$

El ajuste de los datos experimentales para la componente horizontal, indican que la pelota cambia su posición de manera uniforme, de acuerdo con la expresión:

$$
x=-(1.50 \pm 0.01)+(146.1 \pm 0.1) t
$$

Figura 6. Movimiento en dos dimensiones. Para esta fotografía se utilizó el estroboscopio con un disco de una sola ranura

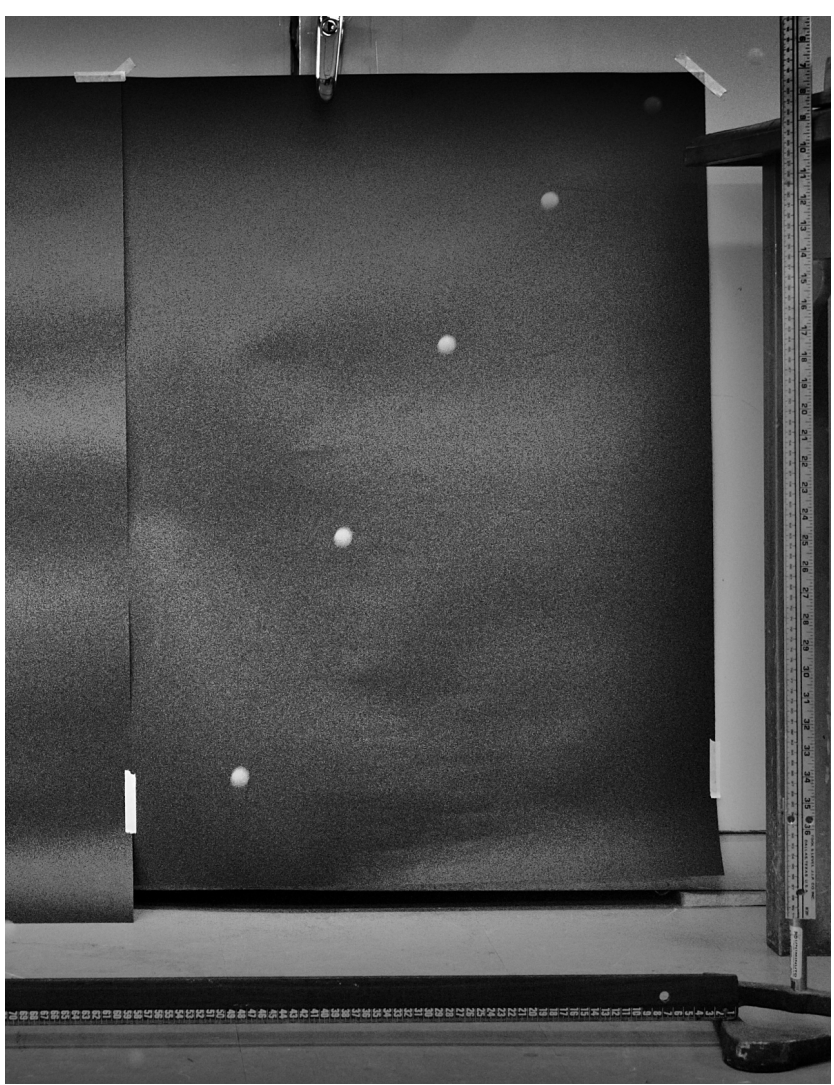

Fuente: adaptación de los autores.

con $x$ en $\mathrm{cm}$ y $t$ en $\mathrm{s}$. En el caso de la componente vertical, la expresión obtenida fue:

$$
y=-(16.4 \pm 0.3)+(39 \pm 3) t-(483 \pm 6) t^{2}
$$

con $y$ en $\mathrm{cm}$ y $t$ en s. De la ec. (5) se obtiene un valor de la gravedad de. Nuevamente, el valor de la aceleración de la gravedad reportado para la ciudad de Bogotá está dentro del rango de incertidumbre. Si bien los valores de la aceleración de la gravedad, obtenidos a partir del análisis de la caída libre y movimiento semiparabólico, contienen dentro de su rango de incertidumbre el valor reportado, la discrepancia entre los resultados experimentales es significativa. Una causa pudo haber sido la dificultad en garantizar un mínimo efecto de paralaje entre la cámara fotográfica y el plano donde se producían los movimientos. 
Figura 7. Movimiento en dos dimensiones. (a) Gráfica de posición horizontal contra tiempo. (b) Gráfica de posición vertical contra tiempo
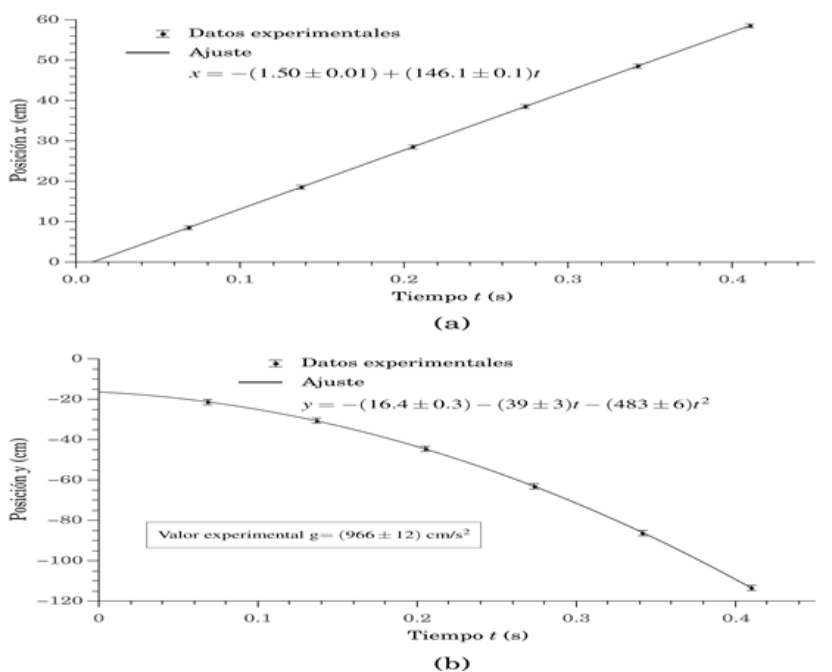

Fuente: adaptación de los autores.

\section{CONCLUSIONES}

La técnica permitió visualizar, por medio de imágenes estroboscópicas obtenidas con una cámara fotográfica, un estroboscopio mecánico y luz ambiente, las posiciones de objetos, mientras se movían en un periodo corto de tiempo.

A partir de las imágenes obtenidas se determinaron las ecuaciones que describen el movimiento en una y dos dimensiones de un carrito de bajo fricción y esferas cayendo, por lo que la técnica de la fotografía estroboscopia sigue siendo un recurso de gran valor didáctico para el trabajo en laboratorios escolares.

El valor encontrado para la aceleración de la gravedad de una bola de golf en caída libre fue de $984 \mathrm{~cm} / \mathrm{s}^{2} \pm 0.8 \%$. El valor obtenido a partir del análisis del movimiento semiparabólico de una esfera fue de $966 \mathrm{~cm} / \mathrm{s}^{2} \pm 1.2 \%$. Ambos valores son consistentes con el valor reportado de $977.39 \mathrm{~cm} / \mathrm{s}^{2}$ para la ciudad de Bogotá, Colombia.

\section{REFERENCIAS}

Chanson, H. (2004). Hydraulics of open channel flow. Butterworth-Heinemann.

Colombo, E.M., Cabrera, M.A., y Medina, C. (1992). El estroboscopio a ranuras y su valor didactico. Caderno Brasileiro de Ensino de Física, 9 (1), 45-53.

Dorling, G. (1966). Stroboscopic photography. Physics Education, 4 (1), 236.

Laws, P., \& Pfister, H. (1998). Using digital video analysis in introductory mechanics projects. The Physics Teacher, 36(5), 282-287.

Medeiros, E., Tavares, O., \& Duarte, S.B. (2009). Inexpensive strobe-like photographs. The Physics Teacher, 47(8), 536-541.

Molina, U., Valbuena, D., y Niebles, F. R. (2006). Fotografiando fenómenos de corta duración de la física. Revista de la Sociedad Colombiana de Física, 38 (2), 545-548.

Ritchie, W. (1966). Methods of teaching dynamics. Physics Education. 3 (1), 185.

Taylor, J. (1997). An Introduction to Error Analysis: The Study of Uncertainties in Physical Measurements. Sausalito, CA, USA.

Valdés, C. R., y Tricio, V. (2007). Actividades experimentales en física general con fotografía y vídeo digitales. Rev. Cub. Física, 24, 46-50. 